\title{
An Isolated Case of Brachyphalangism of the Basal Finger Bones of the Little Finger with Symptoms of Tenosynovitis: A Case Report
}

\author{
Kazuhiko Hashimoto*, Ryosuke Kakinoki, Yukiko Hara, Naohiro Oka, \\ Hiroki Tanaka, Kazuhiro Ohtani, Masao Akagi \\ Department of Orthopedic Surgery, Kindai University Hospital, Osakasayama City, Osaka, Japan \\ Email: *hazzhiko@med.kindai.ac.jp
}

How to cite this paper: Hashimoto, K., Kakinoki, R., Hara, Y., Oka, N., Tanaka, H., Ohtani, K. and Akagi, M. (2018) An Isolated Case of Brachyphalangism of the Basal Finger Bones of the Little Finger with Symptoms of Tenosynovitis: A Case Report. Open Journal of Rheumatology and Autoimmune Diseases, 8, 66-70.

https://doi.org/10.4236/ojra.2018.82006

Received: April 7, 2018

Accepted: May 22, 2018

Published: May 25, 2018

Copyright (ㅇ 2018 by authors and Scientific Research Publishing Inc. This work is licensed under the Creative Commons Attribution International License (CC BY 4.0).

http://creativecommons.org/licenses/by/4.0/

\begin{abstract}
Brachydactyly is a general term that refers to disproportionately short fingers and toes and forms a part of the group of limb malformations characterized by bone dysostosis. Brachydactyly usually occurs either as an isolated malformation or as a part of a complex malformation syndrome. Brachydactyly is classified as types A, B, C, D, or E; brachymetatarsus IV; Sugarman brachydactyly; or Kirner deformity. Various types of isolated brachydactyly are rare, except for types A3 and D. We describe a 15-year-old girl with isolated brachyphalangism of the basal finger bones of the little finger with symptoms of tenosynovitis. Tenosynovitis might be caused by growth deviation between the flexor digitorum superficialis and the flexor digitorum profundus. The patient responded very well to surgical treatment.
\end{abstract}

\section{Keywords}

Brachydactyly, Basal Finger Bones, Tenosynovitis, Proximal Interphalangeal Joint

\section{Introduction}

Brachydactyly is referred to as "short digits" and is characterized by dysostosis [1]. To date, various forms of brachydactyly have been identified. Brachydactyly may also be accompanied by other hand malformations, such as syndactyly, polydactyly, reduction defects, or symphalangism. Isolated brachydactyly has also been reported [2]. Many types of isolated brachydactyly, except for types A3 and $\mathrm{D}$, are rare. Subtle changes elsewhere may appear as isolated brachydactyly. However, isolated brachydactyly with tenosynovitis has not been previously 
reported. We herein present a 15-year-old girl with isolated brachyphalangism of the middle phalangeal bone of the little finger, who demonstrated tenosynovitis-like symptoms that were successfully resolved by surgical intervention. To the best of our knowledge, this is the first case of isolated brachyphalangism presenting tenosynovitis-like symptoms.

\section{Case Presentation}

A 15-year-old girl presented with developing pain and snapping in the left little finger for 1 year prior to our consultation. She has no previous medical history or traumatic history. Her little finger length was almost equal to the proximal interphalangeal (PIP) joint level of the adjacent ring finger. We also observed tenderness and snapping around the PIP joint of the finger (Figure 1(a) and Figure 1(b)). The range of motion of PIP was from 0 to 100 degrees. When she extend her little finger she noticed snapping. The middle phalanges were smaller relative to the proximal and distal phalanges in the bilateral little fingers on plain radiography (Figure 1(c)). Magnetic resonance imaging short-tau inversion recovery revealed high brightness area along the flexor tendon sheath just proximal to the PIP joint level (Figure 1(d)).

We diagnosed tenosynovitis at the A3 pulley accompanied by brachyphalangism and then performed surgery. Through the palmar skin incision of the little finger, the A3 pulley was assessed. When the A3 pulley was resected, a hyperplastic flexor digitorum profundus (FDP) tendon was adherent to the chiasma of the FDS tendon and restricted the FDS motion (Figure 2(a) and Figure 2(b)). A 3-cm-long ulnar half of the FDS tendon was removed from the chiasma to the palmar level (Figure 2(c)). After resection of a cord-like scar on the radial side of the FDS (Figure 2(d)), the snapping was resolved completely (Figure 2(e)). The removed cord-like scar tissue seemed to be scarred vincula (Figure 2(f)). There was no recurrence of the symptoms 1 year postoperatively.

The patient' parents provided consent for publication of this report.

\section{Discussion}

Brachyphalangism often develops as a part of multiple malformations [1]. Isolated brachyphalangism was first reported by Bell in 1951 [3]. Isolated brachydactyly is one of the 10 categories of hand malformations described by Temtamy and McKusick [4]. However, brachydactyly with tenosynovitis has not been previously reported. There are many types of brachydactyly [1], which sometimes presents with various general malformations [1]. It is often difficult to confirm the diagnosis, yet this is important as the treatment varies in accordance with the type of treatment [1]. Therefore, possible cases of brachydactyly should be carefully examined. To our knowledge, we are the first to report an isolated case of brachyphalangism of the little finger with tenosynovitis.

Bell initiated a classification of brachydactyly in 1951 [1], and Temtamy and McKusick further completed it in 1978 [4]. This classification consists of five 

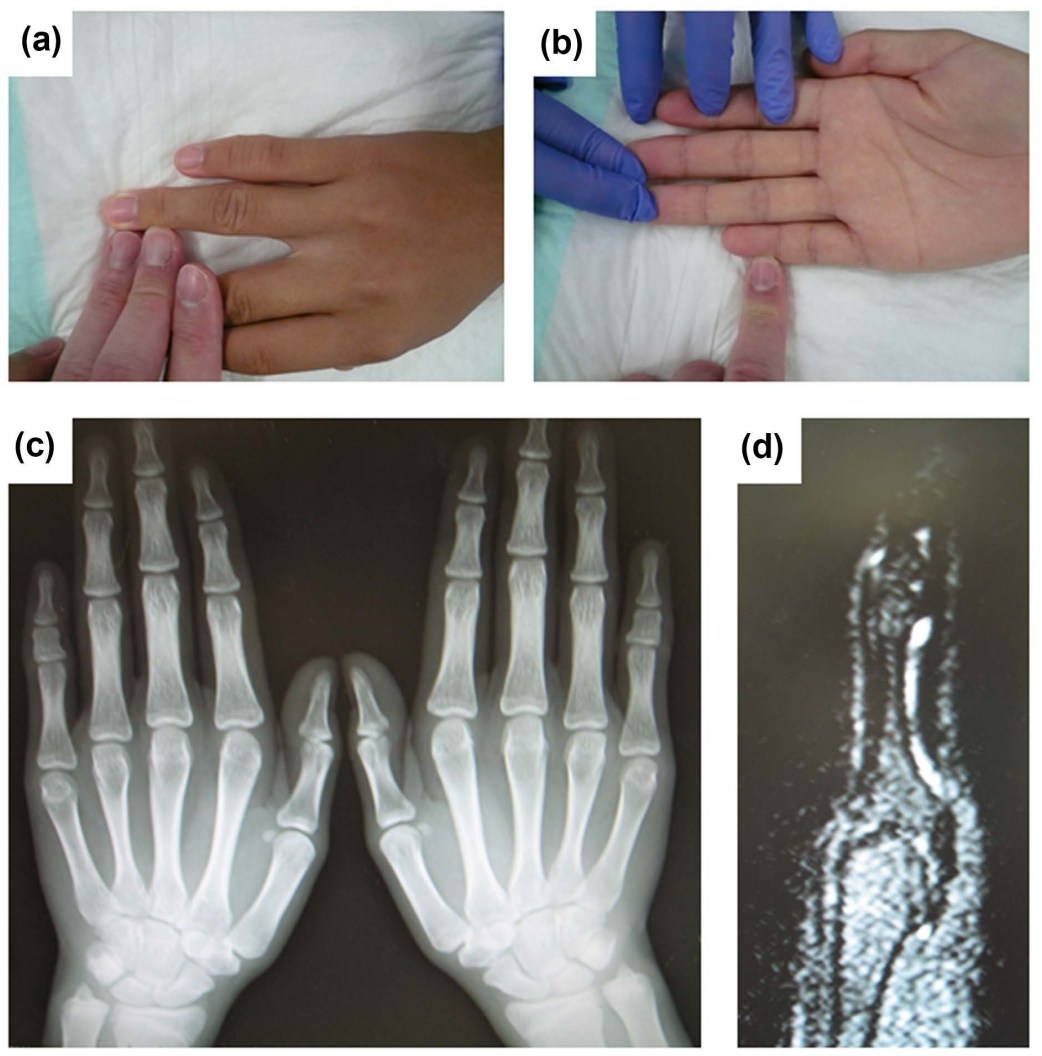

Figure 1. The appearance of the patient's hand (a) and (b). The little finger was shorter than the PIP joint of the ring finger. Radiographic image of both hands (b). Sagittal section of magnetic resonance imaging short-tau inversion recovery of the little finger (d). High brightness intensity was observed in the palm side of the middle phalanx.

individualized types, A to E, and four subgroups, A1 to A4. These malformations are rare, except for types $\mathrm{A} 3$ and $\mathrm{D}$, which occur more frequently and for which the prevalence reaches $2 \%$ [4]. The representative features of each type of deformity are described below [1]. Type A describes cases in which shortening is confined to the middle phalanges, depending on the affected digits. Cases with type B show the feature of absence or hypoplasia of the terminal parts of the index to little fingers. The thumbs are always intact, but frequently show flattening, splitting, or duplication of the distal phalanges. Type $\mathrm{C}$ is a type of hand deformity characterized by brachymesophalangy of the index, middle, and little fingers with hyperphalangy of the index and middle finger, and shortening of the first metacarpal. The ring finger is usually the longest digit. Type $\mathrm{D}$ is the characteristic condition in which the distal phalanx of the thumb alone is shortened. There are various degrees of thumb shortening, either unilaterally or bilaterally. It has been noted that the base of the distal phalanx is broader than the surface of the proximal phalanx to which it articulates, and that the distal end of the bone often shows some hyperplasia. Cases involving type E exhibit shortening of the metatarsals that results from hypoplastic and partially fused metacarpal epiphyses, which is visible on radiographs. Additionally, the terminal 

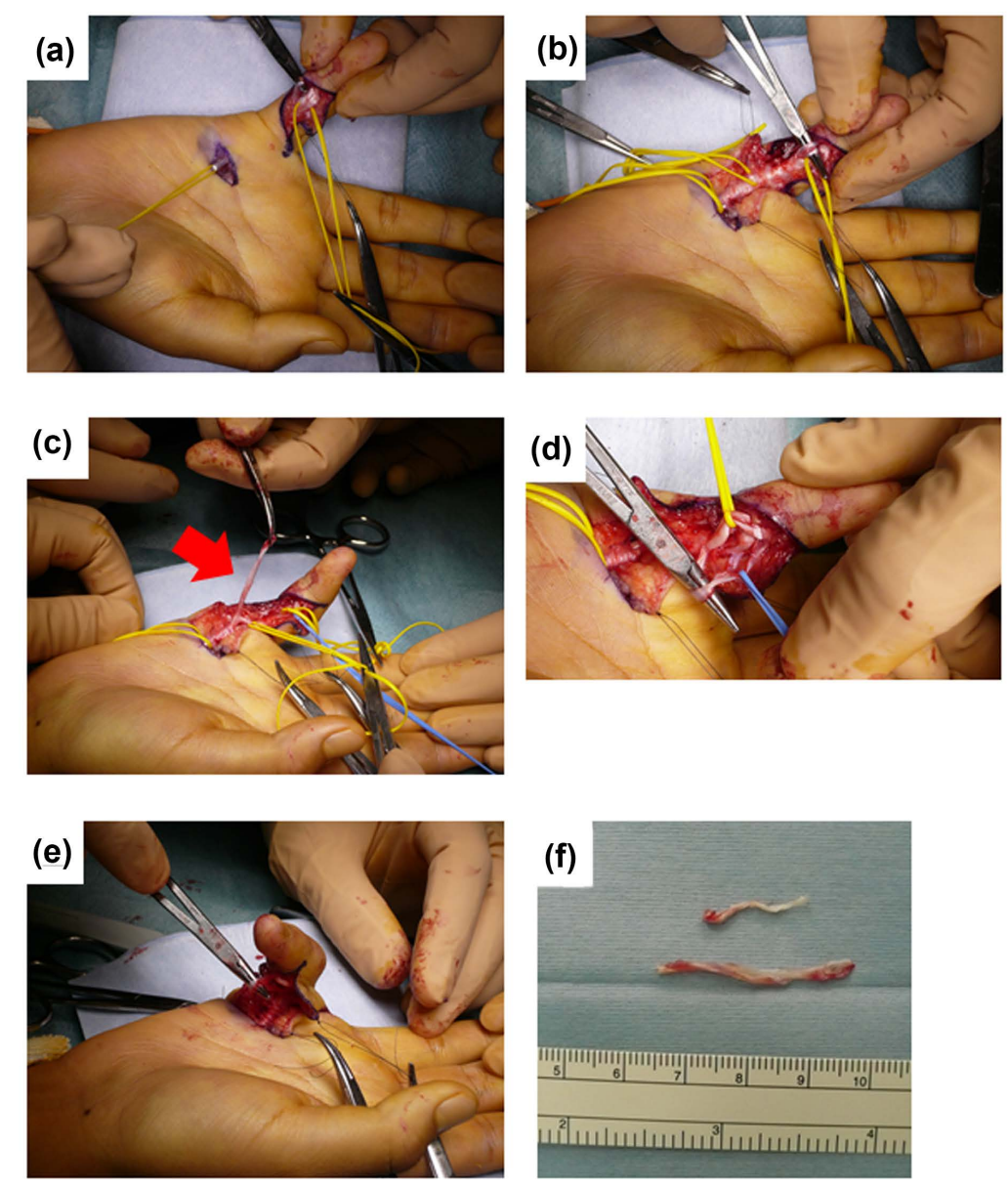

Figure 2. The sliding of the flexor digitorum superficialis (FDS) was restricted (a) and (b). The resected ulnar side of the FDS (c). The vincula-like scar cord at the radial side of FDS (d). The sliding of the FDS is good; thus, the finger showed good flexion and range of motion (e). The resected tissue (f). The upper tissue is the scar. The lower tissue is the ulnar side of the FDS.

phalanges are often short. The current caseis considered type A3. The FDS attached to the middle phalanx is considered a minor malformation, along with type A3 brachyphalangism. Mutation in the COMP gene is reportedly found in brachydactyly and pseudoachondroplasia (PSACH) [5]. In addition, the COMP gene is related to the hyperplasia of soft tissue such as tendons or synovial membranes [6]. Recently, a PSACH case presenting painful locking of the wrist with the COMP gene mutation has been reported [7]. In the current case, we could not examine those gene mutations. This is the limitation of the present study. In that case, the wrist locking was caused by hyperplasia of the extensor carpi radialis longus and brevis tendons. In the present case, hyperplasia of the FDP tendon was observed intraoperatively. FDP hyperplasia was caused by the disproportionate size between the FDP tendon and the FDS tendon chiasma, which might have caused inflammation and scar formation around the chiasma, leading to locking and painful little finger of our patient. Unfortunately, we could not obtain a photo of the hand after surgery. This is a limitation of the 
present study. We confirmed that the diagnosis of the present case is type A3 of brachyphalangism with symptoms of tenosynovitis. We treated the patient with surgical excision on the ulnar side of the FDS and the cord-like scar tissue and achieved favorable outcomes. In her right finger, she had no symptom or complain. We clarify the reason, but snapping or tenderness like as her left finger might occur.

\section{Conclusion}

We have treated the patient of an Isolated brachyphalangism of the basal finger bones of the little finger with symptoms of tenosynovitis with surgical approach. All hand surgeons should keep in mind that isolated brachydactyly might develop tenosynovitis-like symptoms.

\section{Acknowledgements}

We would like to thank Editage (https://www.editage.jp/) for English language editing.

\section{References}

[1] Temtamy, S.A. and Aglan, M.S. (2008) Brachydactyly. Orphanet Journal of Rare Diseases, 3, 15. https://doi.org/10.1186/1750-1172-3-15

[2] David, A., Vincent, M., Quéré, M.P., Lefrançois, T., Frampas, E. and David, A. (2015) Isolated and Syndromic Brachydactylies: Diagnostic Value of Hand X-Rays. Diagnostic and Interventional Imaging, 9, 443-448. https://doi.org/10.1016/j.diii.2014.12.007

[3] Bell, J. (1951) On Brachydactyly and Symphalangism. In: Penrose, L.S., Ed., Treasury of Human Inheritance, Cambridge University Press, London, Volume 5, 191-201.

[4] Temtamy, S.A. and McKusick, V.A. (1978) The Genetics of Hand Malformations. Alan R Liss, Inc., New York, 187-299.

[5] Rock, M.J., Holden, P., Horton, W.A. and Cohn, D.H. (2010) Cartilage Oligomeric Matrix Protein Promotes Cell Attachment via Two Independent Mechanisms Involving CD47 and alphaVbeta3 Integrin. Molecular and Cellular Biochemistry, 338, 215-224. https://doi.org/10.1007/s11010-009-0355-3

[6] Smith, R.K., Zunino, L., Webbon, P.M. and Heinegård, D. (1997) The Distribution of Cartilage Oligomeric Matrix Protein (COMP) in Tendon and Its Variation with tendon Site, Age and Load. Matrix Biology, 16, 255-271. https://doi.org/10.1016/S0945-053X(97)90014-7

[7] Ideta, H., Uchiyama, S., Hayashi, M., Kosho, T., Nakamura, Y. and Kato, H. (2017) Painful Locking of the Wrist in a Patient with Pseudoachondroplasia Confirmed by COMP Mutation. Journal of Surgical Case Reports, 2017, rjw216.

https://doi.org/10.1093/jscr/rjw216 